\title{
Residual $\beta$ cell function and monogenic variants in long-duration type 1 diabetes patients
}

\author{
Marc Gregory Yu, ${ }^{1,2}$ Hillary A. Keenan, ${ }^{1,2}$ Hetal S. Shah, ${ }^{1,2}$ Scott G. Frodsham, ${ }^{3}$ David Pober, ${ }^{1}$ Zhiheng He, ${ }^{1,2}$ Emily A. Wolfson, ${ }^{1}$ \\ Stephanie D’Eon, ${ }^{1}$ Liane J. Tinsley, ${ }^{4}$ Susan Bonner-Weir, ${ }^{1,2}$ Marcus G. Pezzolesi, ${ }^{1,2,3}$ and George Liang King ${ }^{1,2}$ \\ ${ }^{1}$ Research Division, Joslin Diabetes Center, Boston, Massachusetts, USA. ${ }^{2}$ Department of Medicine, Harvard Medical School, Boston, Massachusetts, USA. ${ }^{3}$ Division of Nephrology and Hypertension, University \\ of Utah, Salt Lake City, Utah, USA. ${ }^{4}$ Clinic Administration, Joslin Diabetes Center, Boston, Massachusetts, USA.
}

BACKGROUND. In the Joslin Medalist Study (Medalists), we determined whether significant associations exist between $\beta$ cell function and pathology and clinical characteristics.

\begin{abstract}
METHODS. Individuals with type 1 diabetes (T1D) for $\mathbf{5 0}$ or more years underwent evaluation including HLA analysis, basal and longitudinal autoantibody (AAb) status, and $\beta$ cell function by a mixed-meal tolerance test (MMTT) and a hyperglycemia/ arginine clamp procedure. Postmortem analysis of pancreases from 68 Medalists was performed. Monogenic diabetes genes were screened for the entire cohort.

RESULTS. Of the 1019 Medalists, $32.4 \%$ retained detectable C-peptide levels (>0.05 ng/mL, median: $0.21 \mathrm{ng} / \mathrm{mL}$ ). In those who underwent a MMTT ( $n=516$ ), 5.8\% responded with a doubling of baseline C-peptide levels. Longitudinally ( $n=181$, median: 4 years), C-peptide levels increased in 12.2\% ( $n=22)$ and decreased in $37 \%(n=67)$ of the Medalists. Among those with repeated MMTTs, 5.4\% (3 of 56) and $16.1 \%$ ( 9 of 56 ) had waxing and waning responses, respectively. Thirty Medalists with baseline C-peptide levels of $0.1 \mathrm{ng} / \mathrm{mL}$ or higher underwent the clamp procedure, with $\mathrm{HLA}^{-} / \mathrm{AAb}^{-}$and $\mathrm{HLA}^{+} / \mathrm{AAb}^{-}$ Medalists being most responsive. Postmortem examination of pancreases from 68 Medalists showed that all had scattered insulin-positive cells; 59 additionally had few insulin-positive cells within a few islets; and 14 additionally had lobes with multiple islets with numerous insulin-positive cells. Genetic analysis revealed that $\mathbf{2 8 0}$ Medalists (27.5\%) had monogenic diabetes variants; in $80(7.9 \%)$ of these Medalists, the variants were classified as "likely pathogenic" (rare exome variant ensemble learner [REVEL] >0.75).
\end{abstract}

CONCLUSION. All Medalists retained insulin-positive $\beta$ cells, with many responding to metabolic stimuli even after $\mathbf{5 0}$ years of T1D. The Medalists were heterogeneous with respect to $\beta$ cell function, and many with HLA+ diabetes risk alleles also had monogenic diabetes variants, indicating the importance of genetic testing for clinically diagnosed T1D.

FUNDING. Funding for this work was provided by the Dianne Nunnally Hoppes Fund; the Beatson Pledge Fund; the NIH, National Institute of Diabetes and Digestive and Kidney Diseases (NIDDK); and the American Diabetes Association (ADA).

\section{Introduction}

Pancreatic $\beta$ cell depletion attributed to autoimmunity is the hallmark of type 1 diabetes (T1D). However, recent studies have shown that residual $\beta$ cells may exist despite very long-duration T1D. Even so, natural history data consisting of a comprehensive characterization of $\beta$ cell function in response to metabolic stimuli and a parallel analysis of HLA and monogenic diabetes genotypes and islet morphology in a large cohort of individuals with long-duration T1D have not been available. In the Joslin Medalist Study (1), we described 411 subjects (Medalists) with a documented history of insulin dependence for 50 or more years and characterized their clinical parameters with the postmortem pancreatic morphology

Conflict of interest: GLK received a research grant from Sanofi-Aventis. Copyright: (c) 2019, American Society for Clinical Investigation.

Submitted: January 19, 2019; Accepted: May 10, 2019; Published: July 2, 2019 Reference information: / Clin Invest. 2019;129(8):3252-3263.

https://doi.org/10.1172/JCl127397. of 9 Medalists. While $29.5 \%$ of the participants were positive for either insulinoma antigen 2 (IA2) or glutamic acid decarboxylase (GAD) autoantibodies (AAbs), the majority had detectable random serum C-peptide levels, which were associated with the finding of insulin-positive $\beta$ cells in all postmortem pancreases (1).

Several other studies have documented both the presence of $\beta$ cells and residual C-peptide in individuals with T1D of shorter (mean: 3 years) duration $(2,3)$. In a cohort of T1D patients evaluated in the Bart's Oxford Study, the presence of AAbs was shown to only decline over a median of 23 years in the $\mathrm{AAb}^{+}$subset $(n=132)$, with endogenous C-peptide secretion detected in $35.4 \%$ of the participants (4). Similarly, C-peptide was also detected in 10\% of another T1D cohort with a longer (31-40 years) disease duration (5). These findings of detectable C-peptide and scattered $\beta$ cells in T1D, despite expected ongoing apoptosis, may imply continuing $\beta$ cell formation and strongly suggest that $\beta$ cell regeneration may be possible (6).

In this report of the expanded Joslin Medalist Study, we conducted a longitudinal evaluation of $\beta$ cell function with metabol- 
Table 1. Overall characteristics of the Medalists $(n=1019)$

\begin{tabular}{|c|c|}
\hline & Median (Q1, Q3) $n=1019$ \\
\hline Sex (male) & $462(45.4 \%)$ \\
\hline Age (yr) & $65(60-70)$ \\
\hline Age at diagnosis (yr) & $11(6-15)$ \\
\hline Duration (yr) & $53(51-57)$ \\
\hline HbA1c (\%) & $7.1(6.6-7.7)$ \\
\hline HbA1c $(\mathrm{mmol} / \mathrm{mol})$ & $54.1(48.6-60.7)$ \\
\hline Daily insulin dose (U/kg) & $0.43(0.34-0.54)$ \\
\hline Total cholesterol (mg/dL) & $158(139-181)$ \\
\hline LDL-C (mg/dL) & $78(65-94)$ \\
\hline HDL-C (mg/dL) & $63(51-78)$ \\
\hline Triglycerides (mg/dL) & $65(50-89)$ \\
\hline $\mathrm{BMI}\left(\mathrm{kg} / \mathrm{m}^{2}\right)$ & $25.6(23.0-28.7)$ \\
\hline eGFR (mL/min/1.73 m²) & $71.2(55.5-86.2)$ \\
\hline $\mathrm{ACR}(\mu \mathrm{g} / \mathrm{mg})$ & $12(6.6-33)$ \\
\hline $\operatorname{CRP}(\mathrm{mg} / \mathrm{L})$ & $0.6(0.2-1.5)$ \\
\hline CVD & $391(40.2 \%)$ \\
\hline PDR (ETDRS > 53) & $429(46.6 \%)$ \\
\hline Neuropathy (MNSI $\geq 2$ ) & $655(70 \%)$ \\
\hline Nephropathy (eGFR $\leq 45 \mathrm{~mL} / \mathrm{min} / 1.73 \mathrm{~m}^{2}$ ) & $127(12.6 \%)$ \\
\hline Detectable C-peptide (>0.05 ng/mL) & $327(32.4 \%)$ \\
\hline Random C-peptide (ng/mL) & $0.05(0.05-0.12)$ \\
\hline DR3 & $567(58.8 \%)$ \\
\hline DR4 & $697(72.3 \%)$ \\
\hline DR3 and DR4 & $351(35.9 \%)$ \\
\hline DR3 or DR4 & $913(93.5 \%)$ \\
\hline $\mathrm{CADG5}^{+}$ & $263(28.1 \%)$ \\
\hline $\mid \mathrm{A} 2^{+}$ & $206(22 \%)$ \\
\hline $\mathrm{CADC6}^{+}$or IA2 ${ }^{+}$ & $408(43.6 \%)$ \\
\hline
\end{tabular}

ETDRS, Early Treatment Diabetic Retinopathy Study; LDL-C, low-density lipoprotein cholesterol; HDL-C, high-density lipoprotein cholesterol; ACR, albumin-creatinine ratio; CVD, cardiovascular disease.

ic stimulation and autoimmune status and correlated the results to the participants' known HLA risk alleles, monogenic diabetes genotypes, and islet morphology.

\section{Results}

\section{Baseline characteristics}

Baseline characteristics of the overall Medalist cohort are summarized in Table 1. Of the 1019 participants, $54.7 \%$ were female. At the first visit, the median age of the participants was 65 years, with a median age at diagnosis of 11 years and a median duration of diabetes of 53 years. Clinically, the Medalists had a median BMI of $25.6 \mathrm{~kg} / \mathrm{m}^{2}$ and a glycated hemoglobin A1c (HbA1c) level of 7.1\%, with 93.5\% possessing DR3 or DR4 risk alleles for T1D. Positivity for IA2 or GAD AAbs was found in $43.6 \%$ of Medalists, whereas plasma C-peptide levels were detectable $(>0.05 \mathrm{ng} /$ $\mathrm{mL}$ ) in $32.4 \%$, with a median value of $0.21 \mathrm{ng} / \mathrm{mL}$. As previously reported, the Medalists had a high median HDL $(63 \mathrm{mg} / \mathrm{dL})$ and a low prevalence of nephropathy (12.6\%). Analysis of Medalists with and without detectable C-peptide showed that the former were older at diagnosis, which corresponded to older chronological age. We observed no difference between the 2 groups for HbA1c,
BMI, lipids, or HLA risk alleles. Although higher in the detectable C-peptide group, median C-reactive protein (CRP) levels were within the healthy reference range $(<1 \mathrm{mg} / \mathrm{L})$ for both groups. $\mathrm{AAb}$ levels had contrasting patterns: more of the participants with undetectable C-peptide had GAD AAbs, whereas those with detectable C-peptide were almost twice as likely to express IA2 AAbs. In terms of complications, the overall prevalence of proliferative diabetic retinopathy (PDR) and kidney disease did not differ between the 2 groups. However, those with detectable C-peptide had a lower median estimated glomerular filtration rate (eGFR) and a greater prevalence of peripheral neuropathy as assessed by the Michigan Neuropathy Survey Instrument (MNSI) (Table 2; MNSI $\geq 2$ ).

\section{Histopathology}

As of October 2018, a total of 68 postmortem pancreases from Medalist participants were available for study. These specimens were categorized according to $\beta$ cell distribution on the basis of C-peptide values closest to the date of pancreas procurement (Figure 1). The clinical characteristics of these Medalists did not differ significantly from those of the rest of the cohort. All pancreases contained scattered singlet or doublet insulin-producing $\beta$ cells in a lobular pattern; a majority $(n=59)$ additionally had a few insulin-positive cells within a few islets; and 14 additionally had lobes with multiple islets with numerous insulin-positive cells. These specimens were labeled as category A, B, or C (Figure 1A). Five pancreases in category $C$ contained amyloid in the insulinrich lobes, although their characteristics did not differ from those of the others in the same category. Overall, C-peptide levels increased, changing from category A to category C (Figure 1B). With regard to clinical characteristics, the Medalists in category A tended to be younger, with a younger age of disease onset and a higher median HbA1c value (Supplemental Table 1). Among the Medalists who donated a pancreas, a larger number of $\mathrm{AAb}^{+}$individuals had detectable C-peptide levels as compared with those who were $\mathrm{AAb}^{-}$. Likewise, a greater number of Medalists with monogenic variants had detectable C-peptide levels compared with those with no monogenic variants.

\section{Longitudinal C-peptide and AAb status}

A total of 181 Medalists returned to the Joslin Diabetes Center (JDC) for a second visit after a median of 4.1 years. The characteristics of these individuals at baseline visit did not differ from the those of the other Medalists. Between visits, $60 \%$ (49 of 81) of the Medalists were found to have changed from having detectable C-peptide levels to undetectable C-peptide levels, with a median change of $-0.21 \mathrm{ng} / \mathrm{mL}$ (Table 3). Interestingly, $9 \%$ (9 of 100) of the participants who originally had undetectable C-peptide levels had an increase to a detectable level by a median of $0.21 \mathrm{ng} / \mathrm{mL}$, well above the interassay coefficient of variability (CV) of the C-peptide assay (2.42\%-3.1\%).

We also observed transitions in AAb profiles. Twenty-six percent (20 of 77) of individuals positive for AAb at the first visit had converted to a negative status on the second visit. Furthermore, $16.5 \%$ (17 of 103) converted from negative AAb status on the first visit to positive AAb status on the second visit, with 3 individuals changing from a positive status for one AAb to a positive status for another (Table 3). We found no correlation 
Table 2. Characteristics of Medalists by C-peptide status $(n=1019)$

\begin{tabular}{|c|c|c|c|}
\hline & $\begin{array}{c}\text { C-peptide } \leq 0.05 \mathrm{ng} / \mathrm{mL} \% \text {; } \\
\text { median (Q1, Q3) } \\
n=681\end{array}$ & $\begin{array}{c}\text { C-peptide >0.05 ng/mL\%; } \\
\text { median }(\mathbf{Q} 1, \mathrm{Q} 3) \\
n=327\end{array}$ & $P$ value \\
\hline Sex (male) & $299(43.9 \%)$ & $160(48.9 \%)$ & 0.13 \\
\hline Age (yr) & $64(60-70)$ & $66(61-71)$ & 0.04 \\
\hline Age at diagnosis (yr) & $10(6-14)$ & $12(7-16)$ & 0.01 \\
\hline Duration (yr) & $53(51-56)$ & $53(51-57)$ & 0.48 \\
\hline HbA1c (\%) & $7.1(6.6-7.7)$ & $7.2(6.7-7.7)$ & 0.44 \\
\hline HbA1c (mmol/mol) & $54.1(48.6-60.7)$ & $55.2(49.7-60.7)$ & 0.44 \\
\hline Daily insulin dose (U/kg) & $0.43(0.34-0.54)$ & $0.43(0.34-0.54)$ & 0.61 \\
\hline Total cholesterol (mg/dL) & $157(138-180)$ & 160 (139-183) & 0.15 \\
\hline LDL-C (mg/dL) & $78(64-94)$ & 80 (66-98) & 0.23 \\
\hline $\mathrm{HDL}-\mathrm{C}(\mathrm{mg} / \mathrm{dL})$ & $62.5(50-77.5)$ & $63(51-80)$ & 0.38 \\
\hline Triglycerides (mg/dL) & $64(51-87.5)$ & $67(49-93)$ & 0.57 \\
\hline BMI $\left(\mathrm{kg} / \mathrm{m}^{2}\right)$ & $25.8(22.8-29)$ & $25.5(23.4-28.2)$ & 0.52 \\
\hline eGFR (mL/min/1.73m²) & $73.8(56.2-87.8)$ & $65(53.9-82.6)$ & $<0.0001$ \\
\hline ACR $(\mu \mathrm{g} / \mathrm{mg})$ & $13(7-35)$ & $11(6.1-28)$ & 0.1 \\
\hline $\operatorname{CRP}(\mathrm{mg} / \mathrm{L})$ & $0.4(0.12-1.2)$ & $0.99(0.47-2.2)$ & $<0.0001$ \\
\hline CVD & $261(40.3 \%)$ & $125(39.4 \%)$ & 0.79 \\
\hline PDR (ETDRS > 53) & $295(48.3 \%)$ & $132(43.3 \%)$ & 0.15 \\
\hline Neuropathy (MNSI $\geq 2$ ) & $412(66.7 \%)$ & $239(76.4 \%)$ & $<0.0001$ \\
\hline Nephropathy (eGFR $\leq 45 \mathrm{~mL} / \mathrm{min} / 1.73 \mathrm{~m}^{2}$ ) & $82(12.1 \%)$ & $44(13.5 \%)$ & 0.52 \\
\hline Random C-peptide (ng/mL) & $0.05(0.05,0.05)$ & $0.21(0.13,0.31)$ & $<0.001$ \\
\hline DR3 & $380(59 \%)$ & $184(58.2 \%)$ & 0.82 \\
\hline DR4 & $454(70.5 \%)$ & $241(76.3 \%)$ & 0.06 \\
\hline DR3 and DR4 & $226(34.9 \%)$ & $124(38.2 \%)$ & 0.32 \\
\hline DR3 or DR4 & $608(93.8 \%)$ & $301(92.6 \%)$ & 0.47 \\
\hline $\mathrm{CADC65}^{+}$ & $195(31.4 \%)$ & $67(21.4 \%)$ & $<0.0001$ \\
\hline $\mid \mathrm{A} 2^{+}$ & $106(17 \%)$ & $100(31.9 \%)$ & $<0.0001$ \\
\hline $\mathrm{CADG5}^{+}$or $\mathrm{IAZ}^{+}$ & $262(66.5 \%)$ & $145(33.5 \%)$ & 0.22 \\
\hline
\end{tabular}

Kruskal-Wallis or $\chi^{2}$ test as appropriate.

Of the Medalists who underwent an MMTT, $10.9 \%$ (56 of 516) repeated the procedure during subsequent visits. Among these, 5.4\% (3 of 56) experienced a waxing MMTT response, whereas 16\% (9 of 56) experienced a waning response. Aside from a lower BMI in those with a waxing response, there were no significant differences in other clinical characteristics among the waxing, waning, and no-change groups. Interestingly, all Medalists in the waxing group had no detectable AAbs at the time of either the first or second test (Supplemental Table 3).

Hyperglycemic clamp with arginine infusion. To further characterize insulin secretion, 30 Medalists with a random C-peptide level of $0.1 \mathrm{ng} /$ $\mathrm{mL}$ or higher underwent a hyperglycemic clamp with arginine infusion. With the response again defined as at least a doubling of the baseline C-peptide level, $46.7 \%$ (14 of 30) of these Medalists were responsive to the hyperglycemic/arginine clamp. Of the 22 individuals who have had undergone a previous MMTT, $40.9 \%$ (9 of 22) responded to hyperglycemia, and 63.6\% (14 of 22) responded to arginine. For the clamp procedure, $87.5 \%$ (7 of 8 ) of the MMTT responders and 50\% (7 of 14) of the MMTT nonresponders

between the shifting Abs and the waxing and waning C-peptide levels (Supplemental Table 2).

\section{$\beta$ Cell function studies}

Mixed-meal tolerance test. Given the presence of insulin-expressing $\beta$ cells in all of the studied Medalist pancreases and the fluctuations in C-peptide levels over time, we performed stimulation studies to evaluate $\beta$ cell responsiveness to hyperglycemia. A total of 516 Medalists completed a mixed-meal tolerance test (MMTT), with response to the MMTT defined as at least a doubling of the baseline C-peptide level. The clinical characteristics of these Medalists did not differ significantly from those of the cohort. Of the Medalists who completed an MMTT, 5.8\% (30 of 516) responded to the test with a median change of $0.35 \mathrm{ng} / \mathrm{mL}$. Between responders and nonresponders, the difference in the average AUC was $0.8 \mathrm{ng} / \mathrm{mL}$ $(P<0.002)$ over a 30-minute interval. The responders were older, had an older age at diagnosis, and had higher baseline C-peptide levels. They also had lower AAb and triglyceride levels as compared with nonresponders. We observed no differences between responders and nonresponders with respect to $\mathrm{HbA1c}, \mathrm{BMI}$, other lipid parameters, or prevalence of complications or high-risk HLA alleles (Table 4). also responded to the clamp (Figure 2, A and B). During the clamp, 3 response phases were noted, with the expected initial and second phase responses to hyperglycemia and arginine. However, the Medalists who responded to either hyperglycemic or arginine stimulation $(n=9)$ experienced a third phase 100-200 minutes after the clamp, without any additional stimuli.

Recognizing that there may be heterogeneity to the causes of T1D, we also categorized the Medalists who had undergone the clamp procedure into 3 groups according to the presence of AAbs and HLA risk alleles (Figure 3). Among those who were $\mathrm{HLA}^{+}$but $\mathrm{AAb}^{-}, 36 \%$ (5 of 14 ) responded to hyperglycemia, whereas $64 \%$ (9 of 14) responded to arginine (Figure $3 \mathrm{~A}$ ). Among those who were both $\mathrm{HLA}^{+}$and $\mathrm{AAb}^{+}$, only 18\% (2 of 11) responded to hyperglycemia and $36 \%$ (4 of 11 ) to arginine (Figure $3 \mathrm{~B}$ ). Finally, among those who were both $\mathrm{HLA}^{-}$and $\mathrm{AAb}^{-}$(probably those with monogenic diabetes of unknown etiology), 2 responded ( $40 \% ; 2$ of 5 ) to both glucose and arginine (Figure $3 \mathrm{C}$ ). The characteristics of these Medalists, by clamp group, are summarized in Supplemental Table 4 , and their characteristics by responsiveness to both the MMTT and clamp procedure are summarized in Supplemental Table 5.

Genetic screen for monogenic diabetes. For the entire cohort ( $n=1018)$, we assessed the presence of variants in known mono- 


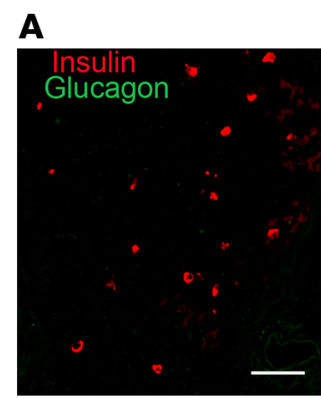

Category A Singlets, outside islets $(68 / 68)$

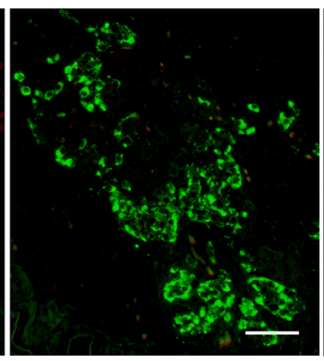

Category $B$

Few within some islets

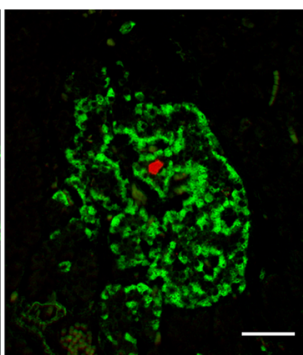

$(59 / 68)$
B

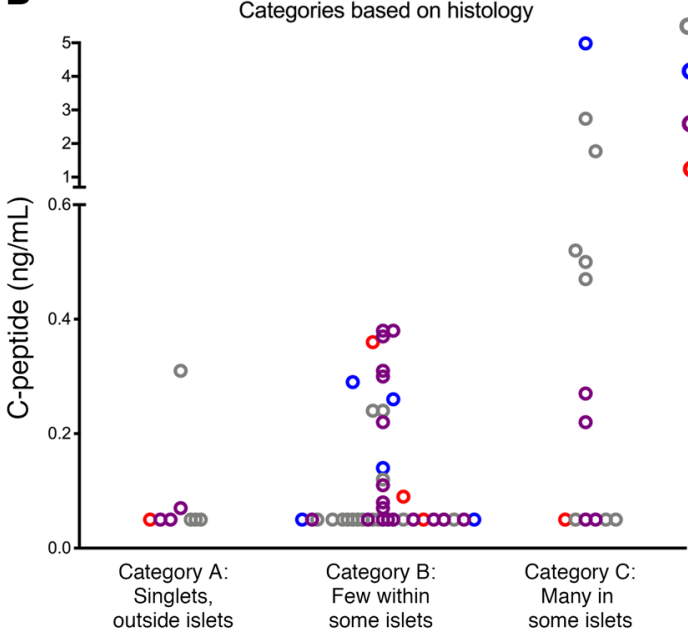

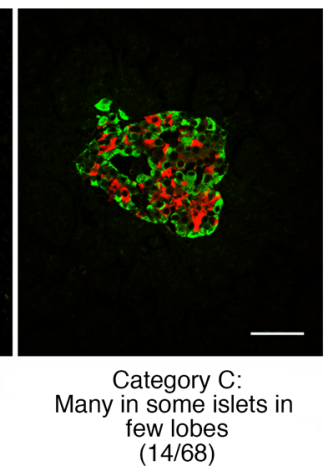

$(14 / 68)$
$\mathrm{AAB}^{-}$
- $\mathrm{AAb}^{-}$monogenic
AAb
$\mathrm{AAb}^{+}$monogenic

Figure 1. Postmortem pancreases from 68 Medalists. (A) All pancreases showed the presence of scattered insulin-positive cells, with some additionally having a few insulin-positive cells within islets and others additionally having lobes with islets with numerous insulin-positive cells. The pancreases were categorized on the basis of distribution of the insulin-positive cells. Insulin (red), glucagon (green). Scale bars: $50 \mu \mathrm{m}$ and $63 \mu \mathrm{m}$ (image on far right). (B) C-peptide levels and AAb presence and monogenic variant within the categories based on distribution of insulin-positive cells in postmortem pancreases $(n=68)$. genic diabetes genes that can affect $\beta$ cell function and found that 280 Medalists (27.5\%) possessed at least 1 rare variant (allele frequencies $<0.1 \%$ ). The ensemble tool REVEL (rare exome variant ensemble learner) was used to predict variant pathogenicity (7). On the basis of a REVEL score cutoff above 0.5 (75.4\% sensitivity, 89.1\% specificity), we identified 151 Medalists (14.8\%) as having likely pathogenic variants. A total of 80 Medalists (7.9\%) carried variants that further satisfied a REVEL cutoff above 0.75 (52.1\% sensitivity, 96.7\% specificity). Supplemental Figure 1 describes the breakdown of likely pathogenic variants, with REVEL scores above 0.75 , and detailed genetic information is described for each variant in Supplemental Table 6 (plus information on the presence of diabetes in 3 generations of these Medalists). Using the REVEL cutoff of greater than 0.75 , Figure 4 shows a comprehensive comparison of median random C-peptide levels among the Medalist subsets. Of those with monogenic variants, the $\mathrm{HLA}^{-}$subjects had significantly higher median random C-peptide levels compared with levels in the $\mathrm{HLA}^{+}$subjects. Within this HLA group, those who were $\mathrm{AAb}^{-}$had significantly higher median random $\mathrm{C}$-peptide levels than did the $\mathrm{AAb}^{+}$group.

Of the 68 Medalists who donated their pancreases, 11 had monogenic diabetes variants, but their pancreases did not differ in the pattern or frequency of insulin-positive cells from those of Medalists without variants. The pancreas of the Medalist with a $C E L$ variant contained overall healthy pancreatic acinar tissue (Supplemental Figure 2). For the Medalists who underwent the clamp procedure, 10 had monogenic diabetes variants. The best responses came from the $\mathrm{HLA}^{-} / \mathrm{AAb}^{-}$individuals, each of whom responded to both the MMTT and clamp procedure. In contrast, only a few of the $\mathrm{HLA}^{+} / \mathrm{AAb}^{+}$subjects responded to either the MMTT or clamp procedure. Table 5 summarizes the clinical characteristics of the Medalists with monogenic diabetes variants who either underwent a clamp or whose pancreas was evaluated postmortem, and their genetic characteristics are summarized in Supplemental Table 7.

For the Medalists who were $\mathrm{HLA}^{+} / \mathrm{AAb}^{-}$and $\mathrm{HLA}^{-} / \mathrm{AAb}^{-}$(those with the best response to metabolic stimulation), the clinical characteristics were further compared between those with monogenic variants and those without (Supplemental Tables 8 and 9). Of the $\mathrm{HLA}^{-} / \mathrm{AAb}^{-}$Medalists, those who had monogenic variants had a higher median C-peptide level than did those without monogenic variants $(0.80 \mathrm{ng} / \mathrm{mL}$ vs. $0.05 \mathrm{ng} / \mathrm{mL}, P=0.0006)$. Moreover, the monogenic group also had a higher proportion of participants with detectable C-peptide levels as compared with the nonmonogenic group (100\% vs. $31.4 \%, P=0.008)$.

\section{Discussion}

This is the first study to our knowledge to document the natural history of $\beta$ cell function and pathology in the same group of individuals with very long-duration T1D. The clinical characteristics of this large cohort showed classical traits of T1D, with a median onset of disease at 11 years of age, normal BMI, and HLA risk alleles in $94 \%$ of these individuals. These findings of the Joslin Medalist Study clearly establish that residual $\beta$ cells are present in 
Table 3. Changes in C-peptide and AAb detectability of Medalists between visits $(n=181)$

\begin{tabular}{cccc}
\multicolumn{5}{c}{ C-peptide detectability } \\
1st visit & 2nd visit & $n(\%)$ & Median change $(\mathrm{ng} / \mathrm{mL})$ \\
- & + & $9 / 100(9 \%)$ & 0.21 \\
+ & - & $49 / 81(60 \%)$ & -0.21 \\
1st visit & 2nd visit & AAb detectability & \\
- & + & $n(\%)$ & \\
+ & - & $20 / 77(26 \%)$ &
\end{tabular}

most, if not all, individuals with T1D, regardless of disease duration or measurable C-peptide levels. In this large Medalist cohort that we followed prospectively for 4 years, approximately $32 \%$ had documented measurable C-peptide levels, yet all 68 studied pancreases were positive for insulin-containing $\beta$ cells. Several studies involving similar cohorts of individuals with T1D, including the Epidemiology of Diabetes Interventions and Complications (EDIC) study and the SEARCH for Diabetes in Youth study, have also reported $20 \%-30 \%$ of participants with detectable C-peptide levels $(3,8-10)$. Moreover, most previous studies of postmortem pancreases from subjects with T1D of varying, but shorter, duration detected $\beta$ cells in $40 \%$ to $88 \%$ of the studied specimens (6, 11-14). Using new ultrasensitive techniques, the recent study by Lam et al. reported that $\beta$ cells with low amounts of insulin were detected in almost all pancreases (14). However, since our study examined the whole pancreas and not just a section or 2, we were able to determine that all subjects had insulin-positive cells using conventional immunofluorescence staining procedures.

Our study focused on individuals with very long-duration T1D and is the only study to our knowledge to show correlations between premortem C-peptide levels and postmortem pancreatic morphological examination in the same patients, in a large sample size. As might be expected, the increasing presence of insulinpositive $\beta$ cells correlated with premortem C-peptide levels, with the most numerous insulin-positive islets generally being detected in subjects with the highest C-peptide levels. However, in a substantial number of subjects, the C-peptide levels did not correlate with the distribution of insulin positivity. In category $\mathrm{A}, 1$ subject only had scattered singlet/doublet insulin-positive cells and yet had high C-peptide levels. Likewise, some had numerous insulinpositive islets (category $\mathrm{C}$ ) and yet had undetectable C-peptide levels. One possible reason for this discrepancy could be the considerable interval (2-11 years) between C-peptide determination and postmortem pancreatic evaluation, such that the $\beta$ cell morphology potentially changed during this specific time frame before death. In contrast, other individuals had C-peptide levels measured only a few days before their death. The huge range of time intervals may have contributed to making exact correlations difficult.

Particularly striking was the lobular distribution of these insulinpositive cells, whether scattered or in islets. It has been known since Gepts' seminal study of patients with recent-onset T1D that $\beta$ cell destruction is lobular, with some lobes bearing pseudoatrophic islets, some having insulitis, and yet others having normal islets and no insulitis $(15,16)$. The reason for this lobular pattern of destruction is still not known. Could the pattern we observed in the Medalists' pancreases be due to protection of some lobes for over 50 years from autoimmunity, or were the $\beta$ cells newly formed since the onset of disease? The presence of scattered insulin-positive cells suggests an ongoing differentiation of new $\beta$ cells rather than replication of preexisting ones. Transdifferentiation from glucagonpositive cells as seen in rodents is unlikely, since the scattered insulin cells are usually not in areas with the very rare scattered glucagon-positive cells; the latter are mainly seen near existing islets (17). Another possibility would be continued pancreatic lobe growth with continued destruction of $\beta$ cells. We would speculate that over the course of a person's lifetime, new small lobes of pancreas are formed with a normal complement of $\beta$ cells but that these cells are then subjected to autoimmune destruction. Previously, we showed both Ki67 within islets as well as apoptotic $\beta$ cells and $\mathrm{CD}^{+}$ lymphocytes in Medalists' pancreases, suggesting the continued turnover of $\beta$ cells (1). The Medalists' pancreases were retrieved only hours after death, so the validity of extensive analysis of these intrapancreatic immune cells is unclear.

Nonetheless, the clear presence of scattered insulin-containing $\beta$ cells in all 68 studied pancreases confirmed the findings of our previous report (1). In the present study, this finding of residual $\beta$ cells in all Medalists' pancreases suggests a preferential survival or regeneration of $\beta$ cells in singlets rather than in islets and further supports the idea of a waxing and waning process of $\beta$ cell remodeling in the presence of autoimmunity. With the identification of persistent proinsulin processing in individuals with undetectable C-peptide, it is intriguing to ask whether other $\beta$ cell populations are dysfunctional enough to be considered merely dormant (18). How the surviving $\beta$ cells managed to elude autoimmune destruction and whether these other groups of dormant $\beta$ cells could be rejuvenated remain important questions (19).

To our knowledge, no other study has examined longitudinal $\mathrm{C}$-peptide and AAb fluctuations in individuals with longstanding T1D. Our Medalist subset tested during at least 2 intervals (with a median of 4 years apart) showed waxing and waning of both $\mathrm{C}$-peptide and AAb levels. Although the overall proportion of participants with detectable C-peptide in our large study fell to $32.4 \%$ from $67.4 \%$, as reported in our 2010 study (1), the transition from undetectable to detectable C-peptide levels in $9 \%$ of the Medalists studied longitudinally is of particular interest. One study of pregnant women with long-duration T1D (median duration: 17 years) reported a median increase of $50 \%$ in C-peptide levels, such that C-peptide became detectable in $97 \%$ of these women at 33 weeks of gestation (20). Another study examining the autopsied pancreases of pregnant women revealed an increased fractional $\beta$ cell area with no change in mean $\beta$ cell size (21). Likewise, both functional changes and increased $\beta$ cell mass have been reported in pregnant rodents (22). The waxing and waning pattern of C-peptide levels suggests that the destructive process for $\beta$ cell loss may also be episodic, as with other systemic autoimmune diseases. In addition, the findings of recurrent high levels of C-peptide in approximately $10 \%$ of individuals with very long-duration T1D clearly establishes the importance of including control groups in interventional studies aimed at preserving or regenerating $\beta$ cells and their function. 
Table 4. Characteristics of Medalists by response to MMTT $(n=516)$

\begin{tabular}{|c|c|c|c|}
\hline & $\begin{array}{c}\text { Responder \%; } \\
\text { Median (Q1, Q3) } \\
n=30\end{array}$ & $\begin{array}{l}\text { Nonresponder \%; } \\
\text { Median }(\mathrm{Q} 1, \mathrm{Q} 3) \\
\quad n=486\end{array}$ & $P$ value \\
\hline Sex (male) & $43.3 \%$ & $45.9 \%$ & 0.79 \\
\hline Age (yr) & $68(63,71)$ & $64(60,69)$ & 0.01 \\
\hline Age at diagnosis (yr) & $16(12,18)$ & $10(6,14)$ & $<0.001$ \\
\hline Duration (yr) & $52(50,53)$ & $52(51,55)$ & 0.34 \\
\hline HbA1c (\%) & $7.1(6.5,7.4)$ & $7.0(6.5,7.6)$ & 0.61 \\
\hline HbA1c (mmol/mol) & $53.6(47.5,57.4)$ & $53.0(47.5,59.6)$ & 0.61 \\
\hline Daily insulin dose (U/kg) & $0.47(0.34,0.61)$ & $0.44(0.34,0.55)$ & 0.51 \\
\hline Total cholesterol (mg/dL) & $156(134,171)$ & $156(136,179)$ & 0.78 \\
\hline LDL-C (mg/dL) & $71[61,88]$ & $77(64,91)$ & 0.51 \\
\hline HDL-C (mg/dL) & $65[49,81]$ & $63(50,79)$ & 0.49 \\
\hline Triglycerides (mg/dL) & $52(44,77)$ & $63[50,83]$ & 0.05 \\
\hline BMI $\left(\mathrm{kg} / \mathrm{m}^{2}\right)$ & $26.4(24.0,28.2)$ & $25.6(23.0,28.9)$ & 0.74 \\
\hline eGFR (mL/min/1.73m²) & $68.6(56.4,80.9)$ & $77.5(62.3,91.0)$ & 0.10 \\
\hline $\mathrm{ACR}(\mu \mathrm{g} / \mathrm{mg})$ & $11.5(5,35.3)$ & $12(6,31)$ & 0.94 \\
\hline $\operatorname{CRP}(\mathrm{mg} / \mathrm{L})$ & $0.24(0.12,1.1)$ & $0.3(0.1,0.9)$ & 0.95 \\
\hline CVD & $36.7 \%$ & $34.7 \%$ & 0.83 \\
\hline PDR (ETDRS >53) & $41.4 \%$ & $46.7 \%$ & 0.79 \\
\hline Neuropathy (MNSI $\geq 2$ ) & $65.5 \%$ & $72.1 \%$ & 0.44 \\
\hline Nephropathy (eGFR $\leq 45 \mathrm{~mL} / \mathrm{min} / 1.73 \mathrm{~m}^{2}$ ) & $6.7 \%$ & $10 \%$ & 0.56 \\
\hline Random C-peptide (ng/mL) & $0.42(0.20,0.91)$ & $0.05(0.05,0.05)$ & $<0.001$ \\
\hline DR3 & $50 \%$ & $58.2 \%$ & 0.39 \\
\hline DR4 & $75 \%$ & $70.1 \%$ & 0.58 \\
\hline DR3 and DR4 & $35.7 \%$ & $34.7 \%$ & 0.92 \\
\hline DR3 or DR4 & $89.3 \%$ & $93.6 \%$ & 0.37 \\
\hline CAD65 $^{+}$ & $24.1 \%$ & $34.8 \%$ & 0.24 \\
\hline $\mathrm{IA}^{+}$ & $13.8 \%$ & $16.6 \%$ & 0.70 \\
\hline $\mathrm{CADG5}^{+}$or $\mathrm{IA2}^{+}$ & $27.6 \%$ & $45.0 \%$ & 0.07 \\
\hline
\end{tabular}

Kruskal-Wallis or $\chi^{2}$ test as appropriate. arginine is preferred when testing the effectiveness of $\beta$ cell function, as it causes a secretory response through membrane depolarization (3, $8,23)$. In our study, arginine stimulation allowed insight into whether the residual $\beta$ cells had lost their glucose responsiveness while still maintaining functionality, as observed in rodents exposed to chronic hyperglycemia.

One striking observation during the clamp procedure was the presence of a third phase of insulin secretion, manifested as a discrete C-peptide spike in those who responded to either hyperglycemic or arginine stimulation. Grodsky described a similar third phase in type 2 diabetes, which he noted as a spontaneous decline in insulin secretion over a 24-hour period of sustained exposure to glucose or other secretagogues. Although the exact mechanisms behind this phenomenon remain unknown, he attributed this effect to either desensitization or waning of priming of threshold-sensitive $\beta$ cells, possibly at a common fundamental location in the secretory site (24). However, to our knowledge, no study has described this phenomenon in patients with T1D. Nevertheless, our functional studies suggested that both scattered singlets and islet-containing $\beta$ cells can respond to physiological stimuli.

Our study is also the first to our knowledge to perform genotyping for monogenic diabetes genes in a large cohort of individuals with longstanding T1D. Overall, genetic analysis revealed rare variants in $27.5 \%$ of the entire cohort. Although some variants, such as the heterozygous EIF2AK3

With regard to AAb fluctuations, studies have provided evidence not only of AAb loss but also of conversion to seropositivity many years after disease onset. In the Bart's Oxford Study, a few participants converted from $\mathrm{GAD}^{-}$to $\mathrm{GAD}^{+}$more than 10 years after diagnosis. However, a similar seroconversion to detectable levels was not seen for other AAbs, and there were no published data on correlations with the C-peptide levels of these participants (4). Despite the shorter disease duration of this cohort, their conversion to detectable GAD levels supports the notion that $\beta$ cell regeneration followed by renewed autoimmune attack may lead to AAb induction. Among the Medalists, a higher proportion of those with decreasing C-peptide levels had AAb positivity at follow-up compared with those whose levels became detectable, suggesting either an active or recent autoimmune attack. Since neither the half-lives of AAbs nor the lifespan of memory cells can be determined in humans, a relationship between these parameters cannot be established without further longitudinal studies with more frequent sampling.

Whatever the cause of residual $\beta$ cells after extreme T1D duration, their consistent presence in all Medalists' pancreases prompted us to undertake a more rigorous evaluation of pancreatic C-peptide secretory ability. Although MMTT and glucagon secretory tests have been used by several groups to determine the secretory ability of residual $\beta$ cell mass in individuals with T1D, mutations, were considered benign, others were classified as likely being pathogenic (7.9\%) using a REVEL cutoff of greater than 0.75 , which provided the highest specificity. There is some controversy surrounding the $B L K$ gene as a cause of monogenic diabetes, as it is not included in the monogenic diabetes panel of the world's largest monogenic diabetes laboratory at the University of Exeter Medical School (25). Among the monogenic variant carriers who underwent the clamp procedure $(n=10)$, the best response was observed in the $\mathrm{HLA}^{-} / \mathrm{AAb}^{-}$group, which also had the highest $\mathrm{C}$-peptide levels compared with levels in their $\mathrm{HLA}^{+} / \mathrm{AAb}^{+}$counterparts. Given the shortcomings of diagnostic evaluations in the past, these $\mathrm{HLA}^{-} /$ $\mathrm{AAb}^{-}$Medalists may have been misdiagnosed with T1D instead of monogenic diabetes. Thus, individuals found to have high levels of C-peptide, despite their longstanding T1D, may benefit from both HLA risk allele evaluation and monogenic diabetes screening, as these may help dictate the ideal course of treatment. To a greater extent, monogenic diabetes screening may benefit individuals with long-duration T1D, regardless of serum C-peptide levels.

A substantial proportion of $\mathrm{HLA}^{+} \mathrm{AAb}^{-}$Medalists also responded to metabolic stimulation, suggesting that this group - despite having high-risk HLA alleles - may likewise benefit from screening for monogenic diabetes. On the other hand, those who were $\mathrm{HLA}^{+}$/ 
A

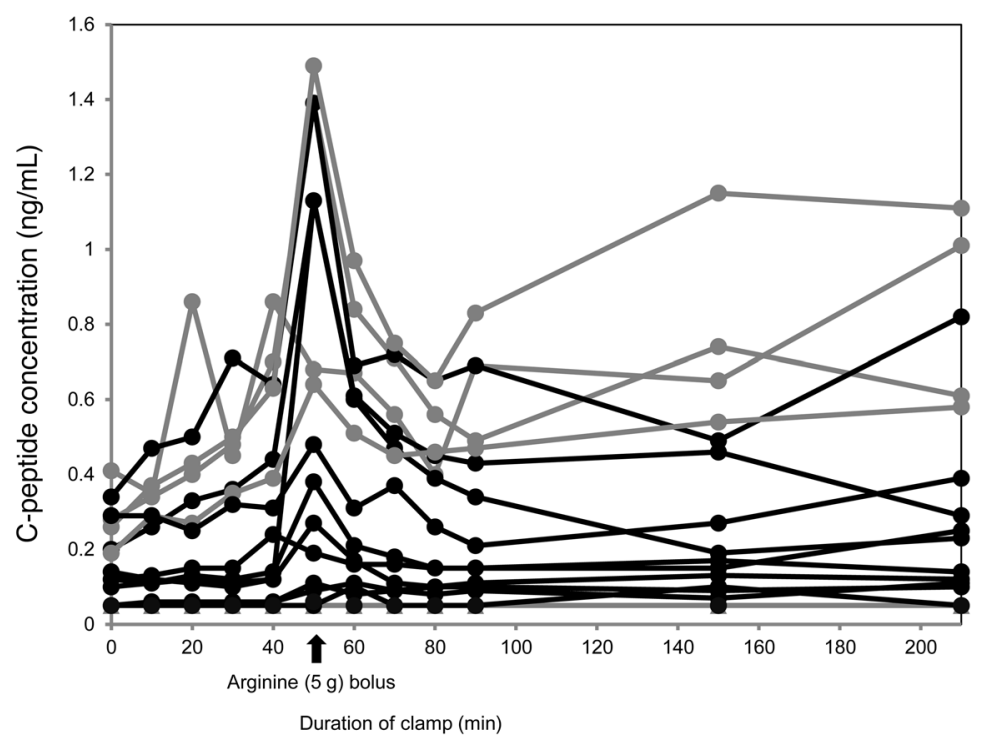

B

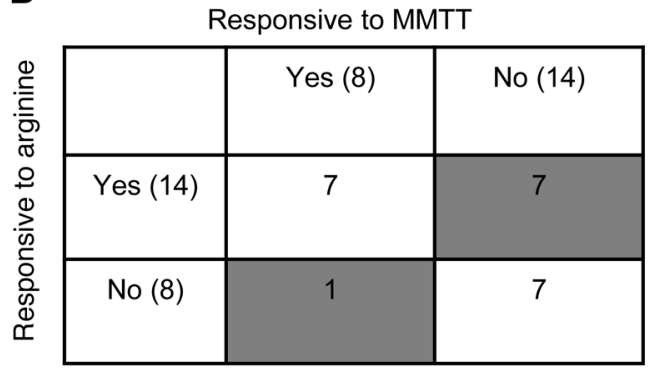

Figure 2. Medalist responses to a hyperglycemic/arginine clamp. (A) Individual responses of the Joslin Medalists to a hyperglycemic clamp with arginine infusion. Blood glucose was clamped at $300 \mathrm{mg} / \mathrm{dL}$ within 45 minutes of beginning the infusion, at which point the subjects received a $5-\mathrm{g}$ arginine bolus. Gray denotes previous MMTT responders; black indicates nonresponders. The graphs of nonresponders may overlap. C-peptide concentration, ng/mL; duration of clamp, $\min (n=22)$. (B) Comparison of the clamp response with MMTT response. Shaded cells represent the cases in which the MMTT and arginine clamp responses were not the same for both tests. The response to the arginine clamp was defined as a doubling of $C$-peptide levels 5 minutes after the arginine bolus infusion; the response to the MMTT was defined as a doubling of baseline C-peptide levels ( $n=22$ ).

$\mathrm{AAb}^{+}$had the least stimulatory response, possibly owing to a greater autoimmune burden. These participants may have harbored "double diabetes" (T1D and monogenic). A literature search revealed only 1 other reported case involving a pediatric patient, who tested positive for both a GCK mutation and islet cell AAbs (26). In general, the exact phenotypic manifestations of such individuals remain unknown, as functional studies of the majority of monogenic diabetes variants are limited. Furthermore, results of the different scoring systems and genetic databases do not always agree with each other, making definitive clinical interpretations difficult. Databases, in particular, while serving as a repository for variants that have been described, still require human judgment in order to obtain an accurate classification. For our study, this included analyzing the participants' clinical characteristics, autoimmune and longitudinal features, and responses to metabolic stimulation.

Likewise, it is important to remember that the genetic analyses performed in this study - including REVEL, functional scoring systems, and database searches - are by no means exhaustive in fully characterizing the pathogenicity of the monogenic variants. However, as our primary focus is to evaluate residual $\beta$ cell function and pathology in long-term T1D, with a correlation between residual C-peptide levels and various factors including the presence of monogenic variants, more detailed genetic characterizations (such as the inclusion of a genetic risk score) are outside the scope of our current work, although they certainly will be important in a follow-up study focused solely on genetics. Furthermore, although $7.9 \%$ of our cohort possessed likely pathogenic monogenic variants, the vast majority of Medalists with detectable C-peptide levels did not carry monogenic variants and also had high-risk HLA alleles. Therefore, we believe that the extent of the genetic analysis already carried out is both a reasonable and sufficient initial analysis to serve this report's purpose, which was to evaluate the potential importance of monogenic variants in C-peptide expression in individuals with long-term T1D.

Nevertheless, the findings from this prospective clinical, genetic, and pathological study clearly show that most individuals with T1D retain insulin-positive $\beta$ cells, with many of these individuals responding to metabolic stimuli even after 50 years of T1D. These individuals, the Medalists, are heterogeneous with respect to $\beta$ cell function and fluctuating course, as well as residual $\beta$ cell morphology, and many with $\mathrm{HLA}^{+}$diabetes risk alleles also have variants in monogenic diabetes genes. These results confirm the benefit of diagnostic genetic screening with therapeutic approaches of $\beta$ cell regeneration in individuals with T1D. Further studies will be needed to determine whether the findings for this group of individuals with long-term T1D can be applicable to those with a shorter duration of T1D.

\section{Methods}

Population. Since 2005, a total of 1019 individuals from all 50 US states have been studied as part of the Joslin's Medalist Study. All participants provided 3 or more forms of documentation demonstrating at least 50 years of T1D. Individuals who received medals between 1998 and 2003 were also sent invitations to participate. Study advertisement was done through word of mouth, clinic postings, and distribution of materials through the American College of Endocrinology, social media, lay publications, and diabetes support groups. In total, $71 \%$ of those who received medals during the study interval participated, with $79 \%$ receiving care outside the JDC. Only individuals who had not undergone pancreas, islet cell, or kidney transplantation were included in the present analysis, as such would confound interpretation of insulin secretory abilities. 

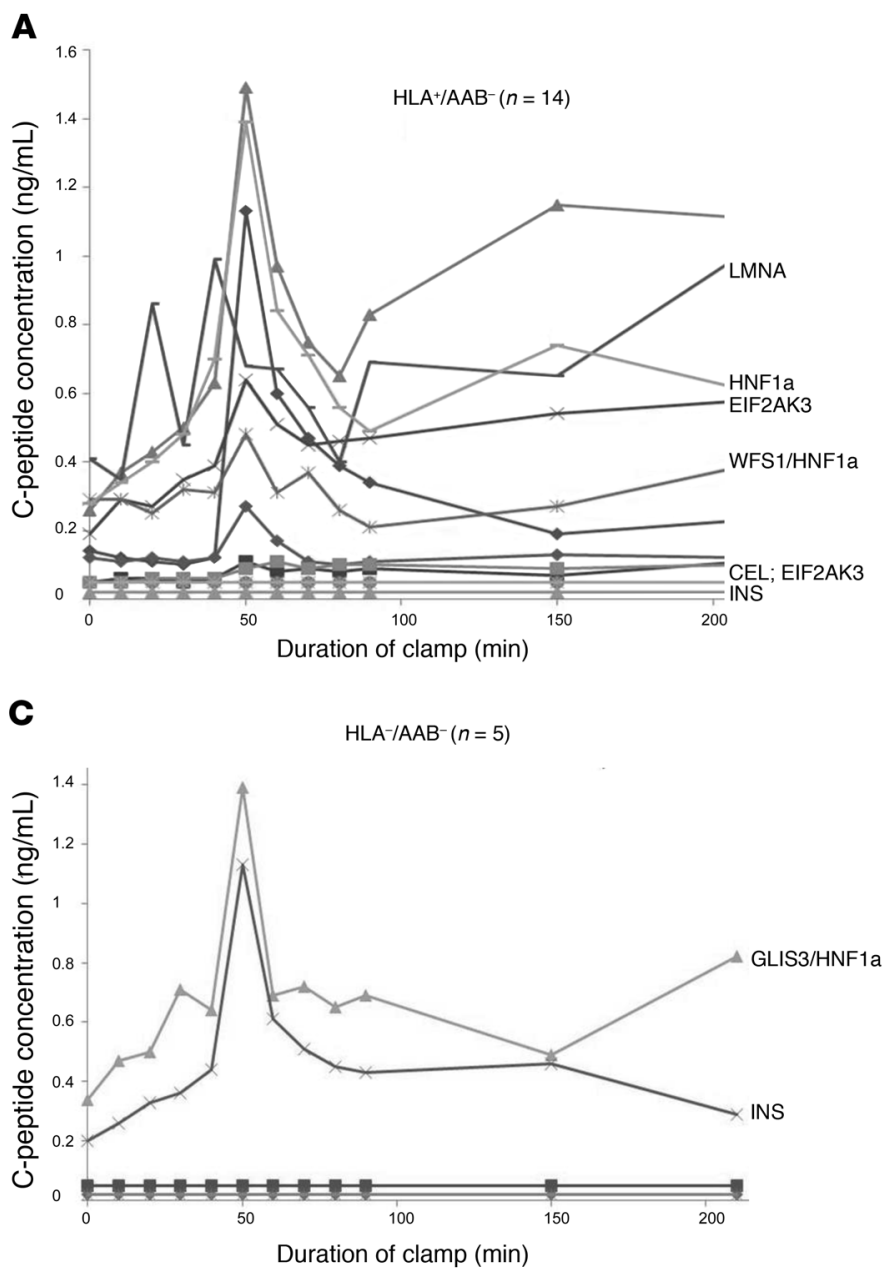

B

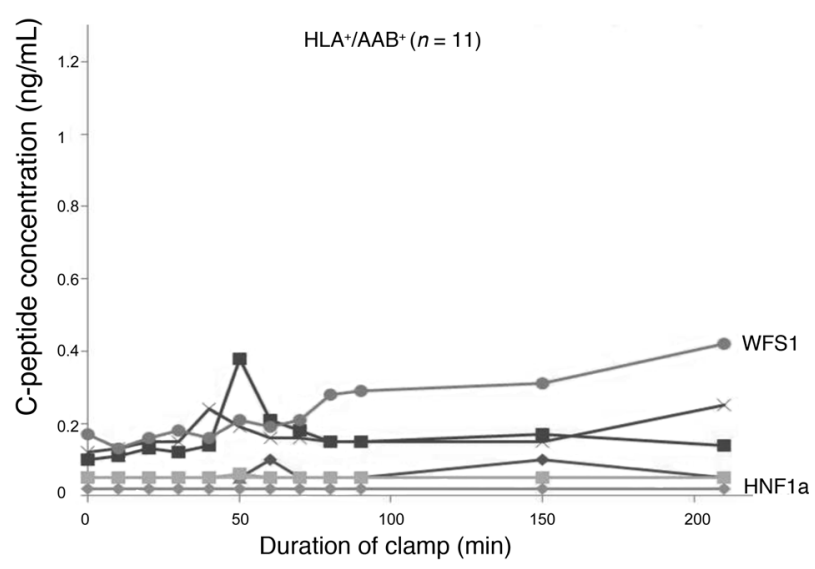

Figure 3. Medalist responses to a hyperglycemic/arginine clamp by risk allele and AAb status. (A) Risk allele $(+), \operatorname{AAb}(-)(n=14)$. (B) Risk-allele and AAb (+) ( $n=11)$. (C) Risk-allele and AAb $(-)(n=5)$. The graphs of nonresponders may overlap. Monogenic variants are indicated on the right. A subset of the data from Figure $2 \mathrm{~A}$ is reshown $(n=22)$. C-peptide concentration, $\mathrm{ng} / \mathrm{mL}$; duration of clamp, min.
Evaluation. Baseline evaluation was done at the JDC by trained staff and involved questionnaires on medical history (including hypoglycemic events and diabetic ketoacidosis [DKA]), physical activity, and nutrition. Samples were drawn to assess biomarkers from serum, plasma, PBMCs, mRNA, DNA, and urine. Assessments for vascular complications (retinopathy, nephropathy, neuropathy, and cardiovascular disease) were also done, the methods of which have been described in detail elsewhere (1) and include the MNSI with monofilament exam, 7-field fundus photography, and eGFR based on serum creatinine $(27,28)$.

Laboratory measures. HbA1c was determined by HPLC (Tosoh G7 and 2.2). Lipid profiles were determined by standard enzymatic methods (Roche Diagnostics, Denka Seiken, and Asahi Kasei). The methods of Yu and colleagues were used at the Joslin Special Assay Core and at the Barbara Davis Center for Childhood Diabetes to assay IA2 and GAD65 AAbs from the baseline blood draws, using a titer cutoff of greater than 5 and greater than 20, respectively (29). Serum C-peptide was determined by radioimmunoassay (Beckman Coulter) and validated at the Northwest Lipid Research Laboratory at the University of Washington, with a detection limit of $0.05 \mathrm{ng} / \mathrm{mL}$ (20).

Genotyping. HLA class II genotyping was performed at the Barbara Davis Center for Childhood Diseases using Luminex xMAP technology (Luminex). Sequence-specific oligonucleotide (SSO) microspheres and PCR reagents used in the assay were obtained from One Lambda Inc. Briefly, the specific HLA region to be typed was amplified by labeling the amplicon with biotin. Amplicon was applied to SSO-coupled microspheres. After incubation, streptavidinphycoerythrin (SAPE) was added to the bead-amplicon mixture. The microspheres were visualized using a Luminex 200 or LabScan3D instrument. The HLA alleles were determined using HLA fusion software (One Lambda Inc.).

Longitudinal visit. Participants were additionally evaluated 3 years after their first visit. As of 2008, the individuals were informed of their eligibility for a second study if it had been at least 3 years since their first visit. Before this time (2005-2008), individuals with C-peptide levels of greater than $0.05 \mathrm{ng} / \mathrm{mL}$ were invited back for an MMTT to validate their original C-peptide value. After 2008, all interested individuals were invited back and underwent the same baseline procedures except for genotyping.

MMTT. Participants were instructed to fast the night before an MMTT. Upon arrival at the JDC, a blood glucose level was measured via glucometer to ensure that the blood glucose level was below $140 \mathrm{mg} / \mathrm{dL}$ before the test was performed. Food or insulin was given to achieve the necessary range. The test began with the participants ingesting a standardized mixed meal of $350 \mathrm{~mL}$ Boost (Nestle Inc.), containing approximately $360 \mathrm{kcal}, 9 \mathrm{~g}$ fat, $50 \mathrm{~g}$ carbohydrate, and $22 \mathrm{~g}$ protein. Blood samples were drawn at baseline and then $30,60,90$, and 120 minutes after the MMTT, and then assayed for C-peptide and glucose levels. 


\section{Table 5. Clinical characteristics of Medalists with monogenic diabetes variants whose pancreas was evaluated postmortem or who underwent the clamp $(n=21)$}

\begin{tabular}{|c|c|c|c|c|c|c|c|c|}
\hline Subject & $\begin{array}{c}\text { Gene } \\
\text { (variant) }\end{array}$ & $\begin{array}{c}\text { HLA } \\
\text { allele }\end{array}$ & AAb & C-peptide & MMTT & Clamp & DKA & Defect \\
\hline 1 & HNF4A (p.1438V) & DR4 & - & + & Not done & Not done & - & Insulin secretion \\
\hline 2 & HNF1A (p.R271W) & DR3 & - & + & + & + & - & Insulin secretion \\
\hline 3 & HNF1A (p.E234X) & - & - & + & + & + & + & Insulin secretion \\
\hline 4 & HNF1A (p.P291S) & DR4 & $\mid A 2$ & + & + & - & - & Insulin secretion \\
\hline 5 & PDX1 (p.P240R) & DR3, DR4 & GAD & - & Not done & Not done & + & Insulin secretion \\
\hline 6 & CEL (p.K4960) & DR3 & - & + & Not done & Not done & - & Insulin secretion \\
\hline 7 & INS (p.G32S) & DR4 & - & + & + & - & - & Insulin secretion \\
\hline 8 & INS (p.L14R) & - & - & + & + & + & + & Insulin secretion \\
\hline 9 & $B L K(p . R 359 C)$ & DR3 & GAD & + & Not done & Not done & - & Insulin secretion \\
\hline 10 & APPL1 (p.I642fs) & DR4 & GAD & - & - & Not done & - & Insulin secretion \\
\hline 11 & APPL1 (p.E6300) & DR3, DR4 & - & - & Not done & Not done & - & Insulin secretion \\
\hline 12 & SLC2A2 (p.R432H) & DR4 & $\mathrm{IA} 2$ & + & Not done & Not done & - & Insulin secretion \\
\hline
\end{tabular}

\begin{tabular}{|c|c|c|c|c|c|c|c|c|}
\hline 13 & SLC2A2 (p.V1011) & DR4 & - & + & Not done & Not done & - & Insulin secretion \\
\hline 14 & $S L C 2 A 2$ (p.R530) & DR4 & IA2, GAD & - & - & Not done & - & Insulin secretion \\
\hline
\end{tabular}

Not done _ - Insulin secretion

$\begin{array}{lllllllll}17 & \text { WFS1 (p.R375C) } & \text { DR3, DR4 } & - & + & + & + & - & \text { Insulin secretion } \\ 18 & \text { LMNA (p.R627H) } & \text { DR3, DR4 } & - & + & - & + & - & \text { Insulin resistance }\end{array}$

\begin{tabular}{|c|c|c|c|c|c|c|c|c|}
\hline 19 & EIF2AK3 (p.P716L) & DR3 & GAD & + & - & + & - & Insulin secretion \\
\hline 20 & $\begin{array}{c}\text { HNF1A (p.R159W), WFS1 } \\
(p . R 375 H)\end{array}$ & DR3 & - & + & - & + & - & Insulin secretion \\
\hline 21 & $\begin{array}{c}\text { CEL (p.1108T), EIF2AK3 } \\
(p . A 422 V)\end{array}$ & DR4 & - & + & + & - & - & Insulin secretion \\
\hline
\end{tabular}

\section{Associated phenotypes in literature}

MODY 1 (39)

MODY 3 (40)

MODY 3 (40)

MODY 3 (40)

MODY 4 (41)

MODY 8 (42)

MODY 10 (43)

MODY 10 (43)

MODY 11 (44)

MODY 14 (45)

MODY 14 (45)

GLUT2 defect and

Fanconi-Bickel

syndrome (46)

GLUT2 defect and

Fanconi-Bickel

syndrome (46)

GLUT2 defect and

Fanconi-Bickel

syndrome (46)

Thiamine-responsive

megaloblastic anemia

(47)

\begin{tabular}{cc}
$\begin{array}{c}\text { Wolfram syndrome } \\
\text { type 1 (48) }\end{array}$ & B \\
$\begin{array}{c}\text { Wolfram syndrome } \\
\text { type 1 (48) }\end{array}$ & Clamp \\
$\begin{array}{c}\text { Familial partial } \\
\text { lipodystrophy } \\
\text { type 2 (49) }\end{array}$ & Clamp \\
$\begin{array}{c}\text { Wolcott-Rallison } \\
\text { syndrome (50) }\end{array}$ & Clamp \\
\hline None reported & Clamp \\
& \\
None reported & Clamp
\end{tabular}

+, response; -, no response (for MMTT and clamp); MODY, maturity-onset diabetes in the young. Additional genetic information including the consensus transcript ID is available in Supplemental Table 7.

Hyperglycemic clamp with arginine stimulation. A hyperglycemic clamp procedure with arginine stimulation was further performed on Medalists with baseline C-peptide levels of $0.1 \mathrm{ng} / \mathrm{mL}$ or higher. Using a case-control design, the participants were categorized into 3 groups on the basis of the presence of high-risk HLA alleles (DR3 and/or DR4) and AAbs: (a) AAb negative and risk-allele positive $(n=14)$; (b) both AAb and risk-allele positive $(n=11)$; and (c) both AAb and risk-allele negative $(n=5)$. The sample size was determined on the basis of detection of a difference in the integrated AUC between the second and third groups, with $80 \%$ power and statistical significance of 0.05 or less.

The test procedure followed a standard protocol. The subjects arrived after a 10-hour fast and followed normal basal insulin and blood glucose monitoring routines. After urine sample collection and vital sign recording, a catheter was placed into the antecubital vein of each arm.
Glucose (D50) was infused at a variable rate throughout the 90-minute protocol to maintain a blood glucose concentration of $300 \mathrm{mg} / \mathrm{dL}$, with infusion of an arginine bolus $(5 \mathrm{~g} / 50 \mathrm{~mL})$ at the 45 -minute mark of the clamp procedure. Blood samples were obtained from the contralateral arm before commencement of the test and every 10 minutes during the clamp procedure, as well as 30,60, and 90 minutes after the clamp procedure to assess glucose, glucagon, and C-peptide concentrations (23).

Histologic studies. Postmortem tissue studies were done on sequentially donated Medalist pancreas of sufficient quality. Premortem consent for organ donation was obtained from donors at the time of study participation, and authorization was obtained from the next of kin at the time of procurement. Logistical arrangements for local procurement were made with the assistance of the National Disease Research Interchange (NDRI). Upon arrival at the JDC, the samples were bread-loafed 


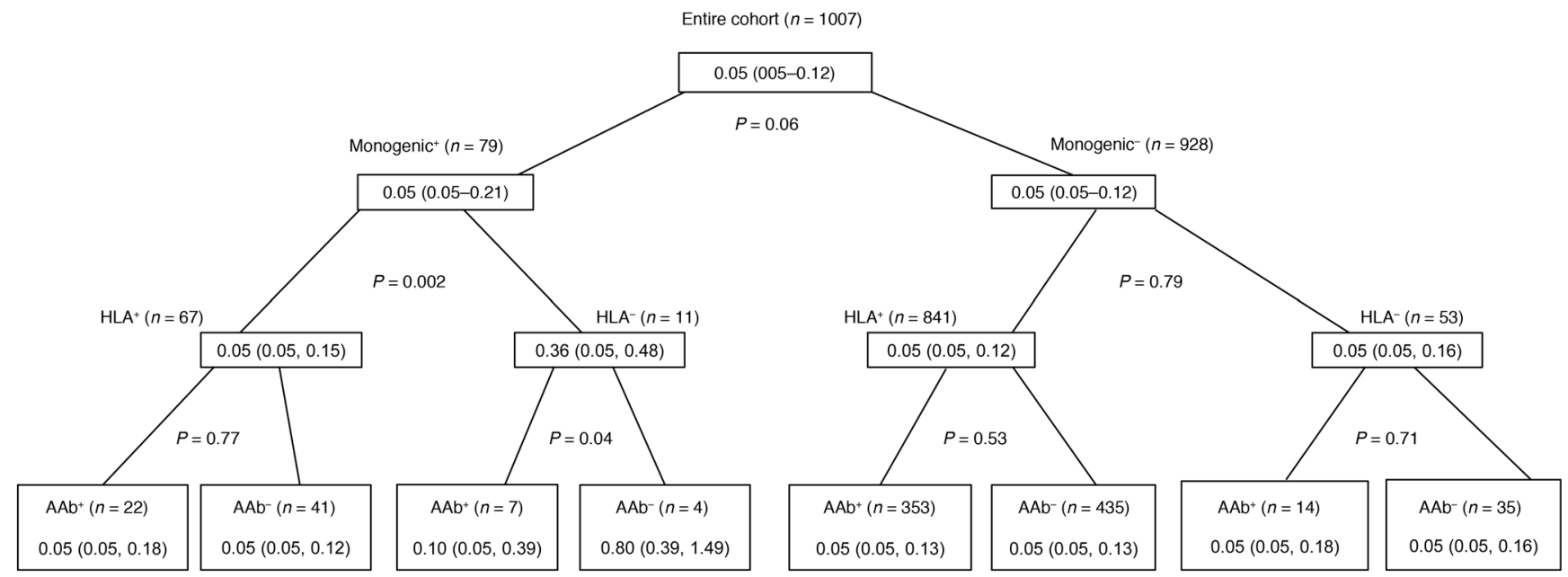

Figure 4. Flowchart comparing the median random C-peptide levels of different subsets in the entire cohort. The REVEL cutoff value was greater than $0.75(n=1007)$. Median (Q1, Q3). A Kruskal-Wallis test was used to determine statistical significance.

into 3 to 30 blocks per pancreas (mean of 15), fixed in $4 \%$ paraformaldehyde, and embedded in paraffin. Paraffin sections were immunostained with guinea pig anti-bovine insulin (1:200, Linco) and rabbit antibovine glucagon (1:2000, M. Appel, University of Massachusetts, Worcester, Massachusetts, USA) and visualized with avidin-biotin complex $(\mathrm{ABC})$ peroxidase for immunofluorescence analysis (1). Images were taken using an Olympus $\mathrm{BH} 2$ microscope or in confocal mode with a Zeiss LSM 710 microscope.

Genetic screen for monogenic diabetes. A genetic screen for rare coding variants in known monogenic diabetes genes was performed on genomic DNA from all Medalists who underwent a hyperglycemic clamp or had postmortem pancreatic analysis using a custom Agilent SureSelect capture assay (Agilent Technologies). This included 13 MODY genes (HNF4A, GCK, HNF1A, PDX1, HNF1B, NEUROD1, KLF11, CEL, PAX4, INS, BLK, ABCC8, and KCNJ11) and 16 neonatal diabetes/familial partial lipodystrophy/familial diabetes genes (PTF1A, NEUROG3, RFX6, EIF2AK3, FOXP3, GLIS3, SLC19A2, SLC2A2, IER3IP1, ZFP57, WFS1, GATA6, GATA4, LMNA, PPARG, and $A P P L 1)$. Samples were fragmented using a Covaris S2 ultrasonicator and prepared for sequencing on an Illumina HiSeq 2500 using a custom DNA library preparation protocol based on the method described by Rohland et al. (30). All coding regions were sequenced to a minimum depth of $75 \times$ coverage. The resulting reads were aligned to the reference human genome (hg19) using Burrows-Wheeler Aligner software (31). The aligned reads were evaluated with SAMtools (32), and variants were identified using the Genome Analysis Toolkit (GATK) (33). To identify potential rare protein-altering variants in monogenic diabetes genes, the resulting variant call format (VCF) file was annotated using ANNOVAR (34) and filtered on the basis of variant function (e.g., nonsynonymous, stop gained, frameshift) and allele frequencies $(<0.1 \%)$ in the Genome Aggregation Database (35). Clinical information on variant pathogenicity was obtained using REVEL and public databases such as ClinVar (36) and InterVar (37), which conform to the 2015 American College of Medical Genetics and Genomics (ACMG) and the Association for Molecular Pathology (AMP) standards and guidelines for the interpretation of sequence variants (38). Compared with other ensemble methods for evaluating variant pathogenicity (MetaLR, MetaSVM, KGGSeq, Condel, Eigen, combined annotation-dependent depletion [CADD], deleterious annotation of genetic variants using neural networks [DANN]), REVEL has a better ability to discriminate Human Gene Mutation Database (HGMD) disease mutations from neutral variants (AUC estimates; REVEL, 0.908 vs. others, $0.730-0.883$ ). It incorporates a total of 18 individual pathogenicity prediction scores from 13 tools as predictive features, with the sensitivity and specificity of each REVEL score threshold determined using out-of-bag predictions for 6182 Human Gene Mutation Database (HGMD) disease mutations and 123,706 putatively neutral exome-sequencing variants (7). In addition, functional scoring systems such as Polyphen2_HDIV, Polyphen2_HVAR, and CADD were used. The first 2, in particular, were chosen as they were shown to have high correlations with REVEL scores (7).

Statistical analysis. The distribution of outcomes and potential covariates were examined for adherence to assumptions of the models used. Descriptive statistics are presented as the median (quartile 1 [Q1], quartile 3 [Q3]) or percentage (n) as appropriate. Tests to determine between-groups differences were conducted using a Wilcoxon rank-sum test or a Kruskal-Wallis test for continuous variables, and a $\chi^{2}$ test of independence or a Fisher's exact test (or test for trend) for categorical variables. For comparing means, a 2-tailed $t$ test was used. Statistical significance was set at a $P$ value of less than 0.05. All statistical analyses were conducted using SAS/STAT software, version 9.4 (SAS Institute).

Study approval. The study was approved by the Committee on Human Studies of the JDC prior to commencement of the study. Written informed consent was obtained from all participants prior to inclusion in the study. All procedures were performed with informed consent and permission of the Committee on Human Studies of the JDC. All participants were systemically deidentified using a numbered code.

\section{Author contributions}

MGY wrote and revised the final manuscript. MGP and SGF performed analysis of monogenic diabetes variants. HSS contributed to revision of the manuscript and data analysis. HAK contributed to the design, implementation, analysis, and writ- 
ing of the manuscript. $\mathrm{ZH}$ contributed to data collection and writing of the manuscript. DP, LJT, and EAW performed statistical analysis. SD contributed to data collection. SBW performed postmortem analysis of pancreases and reviewed and contributed to writing of the manuscript. GLK wrote and reviewed the manuscript and supervised the study.

\section{Acknowledgments}

We wish to thank Gordon Weir, Brooke Sullivan, Jennifer HollisterLock, and Vaja Tchipashvili of the Section on Islet Cell and Regenerative Biology at the JDC, as well as Allison Goldfine and Elena Toschi of the Joslin Clinical Research Center (CRC). This research was funded by the Dianne Nunnally Hoppes Fund; the Beatson Pledge Fund; the
NIH-NIDDK (1DP3DK112192-01 and 1DP3DK094333-01); and the ADA (9-18-CVD1-005, to MGY).

Address correspondence to: George Liang King, One Joslin Place, Boston, Massachusetts 02215, Phone: 617.309.2622; Email: george. king@joslin.harvard.edu. Or to: Marcus G. Pezzolesi, 26 North Medical Drive, Salt Lake City, Utah 84132. Phone: 801.587.7765; Email:marcus.pezzolesi@hsc.utah.edu.

SD's present address is: Voyager Therapeutics Inc., Cambridge, Massachusetts, USA. HK's present address is: Sanofi-Genzyme, Cambridge, Massachusetts, USA. ZH's present address is: Cambridge Health Alliance, Cambridge, Massachusetts, USA.
1. Keenan HA, et al. Residual insulin production and pancreatic $\beta$-cell turnover after 50 years of diabetes: Joslin Medalist Study. Diabetes. 2010;59(11):2846-2853.

2. Roep BO, et al. Auto- and alloimmune reactivity to human islet allografts transplanted into type 1 diabetic patients. Diabetes. 1999;48(3):484-490.

3. Greenbaum CJ, et al. Preservation of beta-cell function in autoantibody-positive youth with diabetes. Diabetes Care. 2009;32(10):1839-1844.

4. Williams GM, et al. Beta cell function and ongoing autoimmunity in long-standing, childhood onset type 1 diabetes. Diabetologia. 2016;59(12):2722-2726

5. Wang L, Lovejoy NF, Faustman DL. Persistence of prolonged C-peptide production in type 1 diabetes as measured with an ultrasensitive C-peptide assay. Diabetes Care. 2012;35(3):465-470.

6. Meier JJ, Bhushan A, Butler AE, Rizza RA, Butler PC. Sustained beta cell apoptosis in patients with long-standing type 1 diabetes: indirect evidence for islet regeneration? Diabetologia. 2005;48(11):2221-2228.

7. Ioannidis NM, et al. REVEL: an ensemble method for predicting the pathogenicity of rare missense variants. Am J Hum Genet. 2016;99(4):877-885.

8. McGee P, et al. Insulin secretion measured by stimulated C-peptide in long-established Type 1 diabetes in the Diabetes Control and Complications Trial (DCCT)/Epidemiology of Diabetes Interventions and Complications (EDIC) cohort: a pilot study. Diabet Med. 2014;31(10):1264-1268.

9. Davis AK, et al. Prevalence of detectable C-Peptide according to age at diagnosis and duration of type 1 diabetes. Diabetes Care. 2015;38(3):476-481.

10. Oram RA, et al. Most people with long-duration type 1 diabetes in a large population-based study are insulin microsecretors. Diabetes Care. 2015;38(2):323-328.

11. Löhr M, Klöppel G. Residual insulin positivity and pancreatic atrophy in relation to duration of chronic type 1 (insulin-dependent) diabetes mellitus and microangiopathy. Diabetologia. 1987;30(10):757-762.

12. Pipeleers D, In't Veld P, Pipeleers-Marichal M, Gorus F. The beta cell population in type 1 diabetes. Novartis Found Symp. 2008;292:19-24; discussion 24 .
13. Md Moin AS, Dhawan S, Shieh C, Butler PC, Cory M, Butler AE. Increased hormone-negative endocrine cells in the pancreas in type 1 diabetes. JClin Endocrinol Metab. 2016;101(9):3487-3496.

14. Lam CJ, Chatterjee A, Shen E, Cox AR, Kushner JA. Low-level insulin content within abundant non- $\beta$ islet endocrine cells in long-standing type 1 diabetes. Diabetes. 2019;68(3):598-608.

15. Gepts W. Pathologic anatomy of the pancreas in juvenile diabetes mellitus. Diabetes. 1965;14(10):619-633.

16. Butler AE, Galasso R, Meier JJ, Basu R, Rizza RA, Butler PC. Modestly increased beta cell apoptosis but no increased beta cell replication in recent-onset type 1 diabetic patients who died of diabetic ketoacidosis. Diabetologia. 2007;50(11):2323-2331.

17. Thorel F, et al. Conversion of adult pancreatic alpha-cells to beta-cells after extreme beta-cell loss. Nature. 2010;464(7292):1149-1154.

18. Sims EK, et al. Proinsulin Secretion Is a Persistent Feature of Type 1 Diabetes. Diabetes Care. 2019;42(2):258-264.

19. Oram RA, Sims EK, Evans-Molina C. Beta cells in type 1 diabetes: mass and function; sleeping or dead? Diabetologia. 2019;62(4):567-577.

20. Nielsen LR, Rehfeld JF, Pedersen-Bjergaard U, Damm P, Mathiesen ER. Pregnancy-induced rise in serum $\mathrm{C}$-peptide concentrations in women with type 1 diabetes. Diabetes Care. 2009;32(6):1052-1057.

21. Butler AE, et al. Adaptive changes in pancreatic beta cell fractional area and beta cell turnover in human pregnancy. Diabetologia. 2010;53(10):2167-2176.

22. Sorenson RL, Brelje TC. Adaptation of islets of Langerhans to pregnancy: beta-cell growth, enhanced insulin secretion and the role of lactogenic hormones. Horm Metab Res. 1997;29(6):301-307.

23. Robertson RP, et al. Assessment of $\beta$-cell mass and $\alpha$ - and $\beta$-cell survival and function by arginine stimulation in human autologous islet recipients. Diabetes. 2015;64(2):565-572.

24. Grodsky GM. A new phase of insulin secretion. How will it contribute to our understanding of beta-cell function? Diabetes. 1989;38(6):673-678.

25. DiabetesGenes. Targeted next generation sequencing: analysis of 61 monogenic diabetes genes. http://www.diabetesgenes.org/tests-for- diabetes-subtypes/targeted-next-generationsequencing-analysis-of-45-monogenic-diabetesgenes. Accessed: April 25, 2019.

26. Concepcion J, and Gottschalk M. MODY2 and Type 1 diabetes in a pediatric patient who developed DKA. Paper presented at: Endocrine Society 2016 Annual Meeting; April 1-4, 2016; Boston, Massachusetts. https://www.endocrine. org/search/\#q=MODY2\%20and\%20Type\%20 1\%20diabetes\%20in\%20a\%20pediatric\%20 patient\%20who\%20developed\%20DKA. Accessed: November 7, 2018.

27. Sun JK, et al. Protection from retinopathy and other complications in patients with type 1 diabetes of extreme duration: the Joslin 50-year medalist study. Diabetes Care. 2011;34(4):968-974.

28. $\mathrm{He} \mathrm{ZH}$, et al. Cardiovascular disease protection in long-duration type 1 diabetes and sex differences. Diabetes Care. 2015;38(5):e73-e74.

29. Yu L, et al. Antiislet autoantibodies usually develop sequentially rather than simultaneously. JClin Endocrinol Metab. 1996;81(12):4264-4267.

30. Rohland N, Reich D. Cost-effective, high-throughput DNA sequencing libraries for multiplexed target capture. Genome Res. 2012;22(5):939-946.

31. Li H, Durbin R. Fast and accurate short read alignment with Burrows-Wheeler transform. Bioinformatics. 2009;25(14):1754-1760.

32. Li H, et al. The Sequence Alignment/ Map format and SAMtools. Bioinformatics. 2009;25(16):2078-2079.

33. McKenna A, et al. The Genome Analysis Toolkit a MapReduce framework for analyzing nextgeneration DNA sequencing data. Genome Res. 2010;20(9):1297-1303.

34. Wang K, Li M, Hakonarson H. ANNOVAR: functional annotation of genetic variants from high-throughput sequencing data. Nucleic Acids Res. 2010;38(16):e164.

35. Broad Institute. Genome Aggregation Database, gnomAD. http://gnomad.broadinstitute.org. Accessed: April 25, 2019.

36. National Center for Biotechnology Information. ClinVar. https://www.ncbi.nlm.nih.gov/clinvar. Accessed: April 25, 2019.

37. Li Q, Wang K. InterVar: clinical interpretation of genetic variants by the 2015 ACMG-AMP Guidelines. Am J Hum Genet. 2017;100(2):267-280.

38. Richards S, et al. Standards and guidelines for 
the interpretation of sequence variants: a joint consensus recommendation of the American College of Medical Genetics and Genomics and the Association for Molecular Pathology. Genet Med. 2015;17(5):405-424.

39. Yamagata K, et al. Mutations in the hepatocyte nuclear factor-4alpha gene in maturity-onset diabetes of the young (MODY1). Nature. 1996;384(6608):458-460.

40. Yamagata K, et al. Mutations in the hepatocyte nuclear factor-1alpha gene in maturityonset diabetes of the young (MODY3). Nature. 1996;384(6608):455-458.

41. Stoffers DA, Zinkin NT, Stanojevic V, Clarke WL, Habener JF. Pancreatic agenesis attributable to a single nucleotide deletion in the human IPF1 gene coding sequence. Nat Genet. 1997;15(1):106-110.
42. Torsvik J, et al. Mutations in the VNTR of the carboxyl-ester lipase gene (CEL) are a rare cause of monogenic diabetes. Hum Genet. 2010;127(1):55-64.

43. Molven A, et al. Mutations in the insulin gene can cause MODY and autoantibody-negative type 1 diabetes. Diabetes. 2008;57(4):1131-1135.

44. Borowiec M, et al. Mutations at the BLK locus linked to maturity onset diabetes of the young and beta-cell dysfunction. Proc Natl Acad Sci U S A. 2009;106(34):14460-14465.

45. Prudente S, et al. Loss-of-function mutations in APPL1 in familial diabetes mellitus. Am J Hum Genet. 2015;97(1):177-185.

46. Santer R, et al. The mutation spectrum of the facilitative glucose transporter gene SLC2A2 (GLUT2) in patients with Fanconi-Bickel syndrome. Hum Genet. 2002;110(1):21-29.
47. Labay V, et al. Mutations in SLC19A2 cause thiamine-responsive megaloblastic anaemia associated with diabetes mellitus and deafness. Nat Genet. 1999;22(3):300-304.

48. Blanco-Aguirre ME, et al. Identification of unsuspected Wolfram syndrome cases through clinical assessment and WFS1 gene screening in type 1 diabetes mellitus patients. Gene. 2015;566(1):63-67.

49. Morel CF, et al. A LMNA splicing mutation in two sisters with severe Dunnigan-type familial partial lipodystrophy type 2.JClin Endocrinol Metab. 2006;91(7):2689-2695.

50. Delépine M, Nicolino M, Barrett T, Golamaully M, Lathrop GM, Julier C. EIF2AK3, encoding translation initiation factor 2-alpha kinase 3, is mutated in patients with Wolcott-Rallison syndrome. Nat Genet. 2000;25(4):406-409. 